\title{
The relationship between the use of cement during total hip replacement and blood pressure values
}

\author{
Pawel Legosz', Anna E. Platek², ${ }^{2}$, Marcin Kotkowski', Bartosz Krzowski', Rafal Kowalczyk', \\ Anna Rys', Karolina Semczuk', Pawel Maldyk', Filip M. Szymanski ${ }^{2}$ \\ 'Department of Orthopaedics and Traumatology of the Musculoskeletal System, Medical University of Warsaw, Warsaw, Poland \\ ${ }^{2} 7^{\text {st }}$ Department of Cardiology, Medical University of Warsaw, Warsaw, Poland \\ ${ }^{3}$ Department of General and Experimental Pathology with Centre for Preclinical Research and Technology (CEPT), \\ Medical University of Warsaw, Warsaw, Poland \\ ${ }^{4}$ Department of Anaesthesiology and Intensive Care, Medical University of Warsaw, Warsaw, Poland
}

\begin{abstract}
Background. Total hip replacement is a common orthopaedic procedure associated with an elevated cardiovascular risk. There are several methods for total hip replacement, including whether or not to use cement for the prosthesis fixation. The aim of the study was to compare clinical characteristics, including blood pressure, in patients undergoing total hip replacement with and without the use of cement.

Material and methods. The study included patients with elective total hip replacement surgery, who were divided into those in whom the procedure was performed with or without the use of medical cement. The criteria for using cement were assessed during operation by the operator, according to the current protocol. All patients were interviewed, screened, and had their medical records checked for the prior diagnosis of cardiovascular risk factors. Blood pressure values were measured before and after the procedure according to the current guidelines.

Results. The study population consisted of 65 patients in whom the total hip replacement was performed (mean age $61.5 \pm 15.0$ years; $50.8 \%$ male). $60 \%$ of patients had the hip replacement without cement and $40.0 \%$ had a procedure with the use of cement. Patients in whom the cement was used were significantly older $(75.0 \pm 8.5$ vs. $53.0 \pm 11.5$ years; $p<0.0001)$ and more often diagnosed with arterial hypertension $(61.5$ vs. $33.3 \% ; p=0.04)$, than those in whom it was not used. Systolic blood pressure values were significantly higher before and after the procedure (138.8 \pm 18.2 vs. $130.8 \pm 21.5 \mathrm{~mm} \mathrm{Hg}, \mathrm{p}=0.04 ; 122.6 \pm 13.3$ vs. $113.8 \pm 14.1 \mathrm{~mm} \mathrm{Hg}, \mathrm{p}=0.03$; respectively) in the group which required cement.

Conclusions: Patients with hip replacement using cement have higher systolic values before, and after the surgery than in patients in whom cement wasn't used. Therefore careful management and risk assessment is especially important in patients receiving the total hip replacement with the use of cement.
\end{abstract}

key words: arterial hypertension, hip replacement, cement

Arterial Hypertens. 2016, vol. 20, no. 3, pages: 119-124

DOI: $10.5603 / A H .2016 .0018$

Address for correspondence: Filip M. Szymanski, MD, PhD

$1^{\text {st }}$ Department of Cardiology,

Medical University of Warsaw, Poland

1 A Banacha Street, 02-097 Warsaw, Poland

tel.: + +48 22 599-19-58; fax: + +48 22 599-19-57

e-mail address: filip.szymanski@wum.edu.pl

V M Copyright $@ 2016$ Via Medica, ISSN 2449-6170 


\section{Introduction}

Total hip replacement is the most common procedure in orthopaedic surgery. All orthopaedic procedures are major surgery procedure associated with perioperative risk $[1,2]$. Proper perioperative cardiovascular risk assessment and management is especially important, because of the high comorbidity rate in total hip replacement patients [3]. There are several methods for total hip replacement. The main distinguisher is the use of cement for the prosthesis. Techniques with cement are preferred when the bone structure has the slower metabolism and is weaker in structure. Based on data from the Polish National Health Fund (Narodowy Fundusz Zdrowia, NFZ) registry during the year 2013, younger patients had mainly non-cemented prosthesis implantation whereas in the group of patients above 80 years of age the number of cement implants was double the number of non-cement prostheses [4]. The aim of the study was to compare clinical characteristics, especially blood pressure values in patients undergoing total hip replacement with and without the use of cement.

\section{Material and methods}

The study was performed with respect to the Declaration of Helsinki and an approval from the Ethics Committee by the Medical University of Warsaw was obtained before the beginning of the study. Every study participant gave a written informed consent prior to the study enrolment.

\section{Study population}

In the study, we enrolled patients who underwent elective total hip replacement surgery. All patients were qualified for the surgery prior to the study beginning. Exclusion criteria were: age $<18$ or $\geq$ 75 years, disqualification from the operation, the absence of written informed consent or contraindications for any of the study protocol examinations. Subsequent patients were divided into those in whom the procedure was performed with or without the use of medical cement. The criteria for using cement were assessed during operation by the operator, according to the current protocol. Investigators had no impact on the kind of procedure that was used.

\section{Diagnosis of cardiovascular risk factors and blood pressure}

All patients were interviewed, screened, and had their medical records checked for the prior diagnosis of cardiovascular risk factors. Diagnosis of the condition was made according to the current guidelines. For example, diagnosis of arterial hypertension was made when office blood pressure was equal or exceeded the values of 140 for systolic blood pressure (SBP) and/or $90 \mathrm{~mm} \mathrm{Hg}$ for diastolic blood pressure (DBP), and/or patient had eligible medical records proofing the previous diagnosis, and/or patient was currently taking medications used for hypertension treatment. All study participants were screened for arterial hypertension. Patients had their blood pressure measured on admission and after the procedure by a qualified physician. The number of required measurements, measure techniques and settings were in accordance with the current guidelines for arterial hypertension diagnosis and management of the $\mathrm{Eu}$ ropean Society of Cardiology and European Society of Hypertension [5].

Blood samples were obtained from all study participants on admission from the cubital vein. Blood was drawn after the standard night-time fasting. All laboratory parameters were immediately assessed in the blood samples according to the current standard in the Hospital Laboratory.

\section{Statistical analysis}

Statistical analysis was performed using the SPSS v 21.0 (SPSS Inc, Chicago, IL, USA). Continuous data are presented as mean \pm standard deviation (SD) and were compared using the Mann-Whitney test or Student's t-test. Categorical variables were compared using either the $\chi^{2}$ or Fisher's exact tests. A p value of less than 0.05 was considered statistically significant, whereas the confidence intervals (CI) were 95\%.

\section{Results}

The study population consisted of 65 patients in whom the total hip replacement was performed. The mean age of the study population was $61.5 \pm 15.0$ years and $50.8 \%$ of patients were male. The cardiovascular risk factor with the highest prevalence in the study population was arterial hypertension observed in $44.6 \%$ of cases. $9.2 \%$ of patients had diabetes mellitus, $6.3 \%$ had coronary artery disease and $4.6 \%$ had diagnosed heart failure. Detailed characteristics of the study population can be found in Table I.

After dividing patients according to the procedure type we found that there we $39(60.0 \%)$ patients with the hip replacement without cement and 26 $(40.0 \%)$ had a procedure with the use of cement. Patients in whom the cement was used were significantly older $(75.0 \pm 8.5$ vs. $53.0 \pm 11.5$ years; 
Table I. Baseline characteristics of the study population

\begin{tabular}{|c|c|}
\hline Parameter & $\begin{array}{c}\text { Value } \\
(\% \text { or mean } \pm S D)\end{array}$ \\
\hline Age (years) & $61.8 \pm 15.0$ \\
\hline Male sex & $33(50.8 \%)$ \\
\hline Heart failure & $3(4.6 \%)$ \\
\hline Diabetes mellitus & $6(9.2 \%)$ \\
\hline Prior stroke or TIA & $2(3.1 \%)$ \\
\hline Prior myocardial infarction & $1(1.6 \%)$ \\
\hline Peripheral artery disease & $1(1.6 \%)$ \\
\hline Smoking & $1(1.6 \%)$ \\
\hline Dyslipidaemia & $1(1.6 \%)$ \\
\hline Coronary artery disease & $4(6.3 \%)$ \\
\hline Asthma or COPD & $1(1.6 \%)$ \\
\hline Height [cm] & $166.2 \pm 22.6$ \\
\hline Weight $[\mathrm{kg}]$ & $76.3 \pm 12.5$ \\
\hline \multicolumn{2}{|c|}{ Blood pressure parameters } \\
\hline Previously diagnosed arterial hypertension & $29(44.6 \%)$ \\
\hline SBP on admission [mm Hg] & $134.3 \pm 20.3$ \\
\hline DBP on admission [mm Hg] & $76.3 \pm 15.4$ \\
\hline HR on admission (beats per minute) & $72.3 \pm 12.6$ \\
\hline SBP after surgery [mm Hg] & $117.6 \pm 14.3$ \\
\hline DBP after surgery [mm $\mathrm{Hg}]$ & $66.6 \pm 10.6$ \\
\hline HR after surgery (beats per minute) & $69.2 \pm 12.9$ \\
\hline \multicolumn{2}{|l|}{ Laboratory results } \\
\hline White blood cells & $7.9 \pm 3.2$ \\
\hline Red blood cells & $4.4 \pm 0.6$ \\
\hline Haemoglobin & $132.5 \pm 18.5$ \\
\hline Haematocrit (\%) & $40.0 \pm 5.2$ \\
\hline Mean cell volume & $91.4 \pm 5.6$ \\
\hline MCHC & $326.4 \pm 40.3$ \\
\hline Platelets & $239.7 \pm 68.4$ \\
\hline Sodium & $140.5 \pm 3.1$ \\
\hline Potassium & $4.2 \pm 0.4$ \\
\hline Glucose & $101.1 \pm 22$ \\
\hline Urea & $37.3 \pm 18.8$ \\
\hline Creatinine & $1.0 \pm 0.4$ \\
\hline INR & $1.0 \pm 0.1$ \\
\hline APTT & $30.1 \pm 3.1$ \\
\hline
\end{tabular}

COPD — chronic, obstructive pulmonary disease, DBP — diastolic blood pressure, $\mathrm{HR}$ - heart rate, $\mathrm{SBP}$ - systolic blood pressure, SD - standard deviation, TIA — transient ischaemic attack Data are presented as number (\%) or mean $\pm S D$

$\mathrm{p}<0.0001)$ than those in whom it was not used. They were also more often diagnosed with arterial hypertension $(61.5$ vs. $33.3 \% ; \mathrm{p}=0.04)$ and prevalence of heart failure was on the edge of statistical significance ( 11.5 vs. $0.0 \% ; \mathrm{p}=0.06)$. When we analysed blood pressure values we found that patients

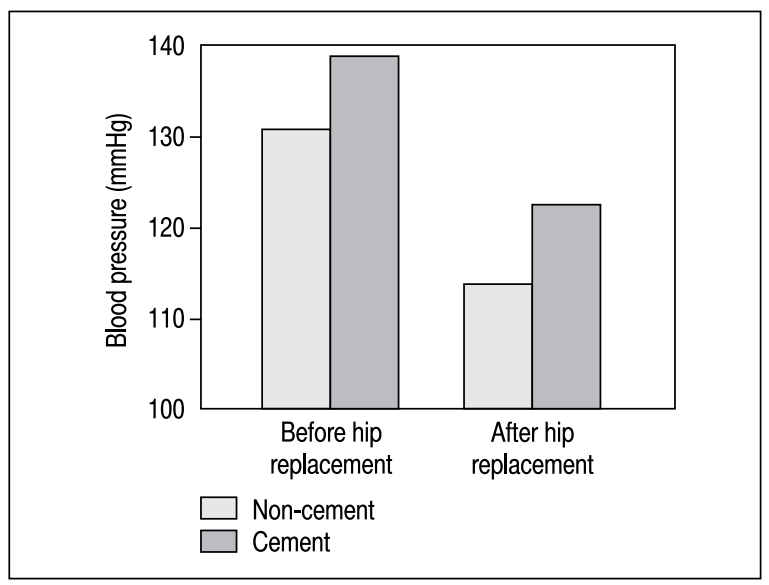

Figure 1. Differences in blood pressure values in patients with and without the use of cement in hip replacement

requiring cement during procedure had significantly higher systolic blood pressure values before and after the procedure $(138.8 \pm 18.2$ vs. $130.8 \pm 21.5 \mathrm{~mm} \mathrm{Hg}$, $\mathrm{p}=0.04 ; 122.6 \pm 13.3$ vs. $113.8 \pm 14.1 \mathrm{~mm} \mathrm{Hg}$, $\mathrm{p}=0.03$; respectively) (Figure 1).

Several, significant differences were also found in the results of laboratory analysis and covered haemoglobin concentration, haematocrit, and urea and creatinine concentration. Details can be found in Table II.

\section{Discussion}

The discovery and production of medical cement date back to the year 1902. In this period, Otto Rohm introduced a method of polymerization of acrylic acid and production of cement (polymethyl methacrylate - PMMA). In the year 1936, industrial production of PMMA began. In that time medical cement was used to fill skull defects/gaps in animals (1939) as well as humans (1940) and in dentistry. During the Second World War, neurosurgeons started using PMMA during cranioplasty [6-8]. In the following years, indications for cement use began to expand. In orthopaedic surgery, bone cement is in use for over 50 years. It is applied in joint replacement surgery (mainly hip, knee, and shoulder) as a fixing agent of the prosthesis with bone but also enables filling of lost bone tissue secondary to removed cancer and in compression fractures of the vertebrae (vertebroplasty). It often supports and supplements other methods of stabilizing a fracture. In arthroplasties, bone cement can contain antibiotics reducing the number of post-surgery infections [9]. Bone cement functions by transferring and evenly distributing any load applied to the bone [10]. In recent years, there 
Table II. Differences between patients undergoing hip replacement with or without cement

\begin{tabular}{|c|c|c|c|}
\hline Parameter & $\begin{array}{l}\text { Patients with hip replacement } \\
\text { without cement }(n=39)\end{array}$ & $\begin{array}{l}\text { Patients with hip replacement with } \\
\text { cement }(n=26)\end{array}$ & $\mathrm{p}$ value \\
\hline Age (years) & $53.0 \pm 11.5$ & $75.0 \pm 8.5$ & $<0.0001$ \\
\hline Male sex & $22(56.4 \%)$ & $11(42.3 \%)$ & 0.32 \\
\hline Heart failure & $0(0.0 \%)$ & $3(11.5 \%)$ & 0.06 \\
\hline Diabetes mellitus & $3(7.7 \%)$ & $3(11.5 \%)$ & 0.45 \\
\hline Prior stroke or TIA & $1(2.6 \%)$ & $1(4.0 \%)$ & 1.00 \\
\hline Prior myocardial infarction & $1(2.6 \%)$ & $0(0.0 \%)$ & 1.00 \\
\hline Peripheral artery disease & $0(0.0 \%)$ & $1(4.2 \%)$ & 0.38 \\
\hline Smoking & $1(2.6 \%)$ & $0(0.0 \%)$ & 1.00 \\
\hline Dyslipidaemia & $1(2.6 \%)$ & $0(0.0 \%)$ & 1.00 \\
\hline Coronary artery disease & $1(2.6 \%)$ & $3(11.5 \%)$ & 0.29 \\
\hline Asthma or COPD & $0(0.0 \%)$ & $1(4.2 \%)$ & 0.40 \\
\hline Height [cm] & $166.3 \pm 2.9$ & $166.1 \pm 8.2$ & 0.19 \\
\hline Weight [kg] & $76.9 \pm 14.5$ & $75.4 \pm 9.1$ & 0.93 \\
\hline \multicolumn{4}{|c|}{ Blood pressure parameters } \\
\hline Previously diagnosed arterial hypertension & $13(33.3 \%)$ & $16(61.5 \%)$ & 0.04 \\
\hline SBP on admission [mm Hg] & $130.8 \pm 21.5$ & $138.8 \pm 18.2$ & 0.04 \\
\hline DBP on admission [mm Hg] & $75.0 \pm 16.7$ & $78.0 \pm 13.6$ & 0.25 \\
\hline HR on admission (beats per minute) & $72.4 \pm 13.2$ & $72.0 \pm 12.0$ & 0.95 \\
\hline SBP after surgery [mm Hg] & $113.8 \pm 14.1$ & $122.6 \pm 13.3$ & 0.03 \\
\hline DBP after surgery [mm Hg] & $66.8 \pm 11.5$ & $66.2 \pm 9.5$ & 0.96 \\
\hline HR after surgery (beats per minute) & $70.2 \pm 14.0$ & $67.8 \pm 11.4$ & 0.82 \\
\hline \multicolumn{4}{|c|}{ Laboratory results } \\
\hline White blood cells & $7.5 \pm 2.4$ & $8.5 \pm 4.0$ & 0.39 \\
\hline Red blood cells & $4.5 \pm 0.7$ & $4.3 \pm 0.4$ & 0.15 \\
\hline Haemoglobin & $136.8 \pm 20.1$ & $126.2 \pm 14.1$ & 0.03 \\
\hline Haematocrit (\%) & $41.0 \pm 5.7$ & $38.4 \pm 4.1$ & 0.04 \\
\hline Mean cell volume & $92.4 \pm 6.3$ & $90.0 \pm 4.0$ & 0.33 \\
\hline $\mathrm{MCHC}$ & $333.4 \pm 8.8$ & $316.4 \pm 61.4$ & 0.03 \\
\hline Platelets & $246.2 \pm 73.3$ & $230.3 \pm 61.1$ & 0.54 \\
\hline Sodium & $139.8 \pm 2.9$ & $141.4 \pm 3.2$ & 0.08 \\
\hline Potassium & $4.2 \pm 0.4$ & $4.2 \pm 0.4$ & 0.75 \\
\hline Glucose & $100.6 \pm 23.6$ & $102.0 \pm 19.7$ & 0.45 \\
\hline Urea & $34.0 \pm 19.9$ & $41.9 \pm 16.5$ & 0.01 \\
\hline Creatinine & $1.0 \pm 0.4$ & $1.1 \pm 0.3$ & 0.06 \\
\hline INR & $1.0 \pm 0.1$ & $1.0 \pm 0.1$ & 0.33 \\
\hline APTT & $30.1 \pm 3.3$ & $30.1 \pm 2.9$ & 0.85 \\
\hline
\end{tabular}

COPD — chronic, obstructive pulmonary disease, DBP — diastolic blood pressure, HR — heart rate, SBP — systolic blood pressure, SD — standard deviation, TIA — transient ischaemic attack Data are presented as number $(\%)$ or mean $\pm S D$

is the debate on the potential for bone cement to cause harm to patients and benefits of non-cemented arthroplasties are being raised. However, considering a certain cohort of patients, fixation of the prosthesis using bone cement still plays a crucial role in achieving satisfactory results after surgery. Examples of total hip replacement with or without cement are shown in Figures 2 and 3, respectively.

Currently, indications and advantages of the cement use can be summarized as follows:

- cement is mostly indicated in older patients (more than 75 years), but are also used in younger 


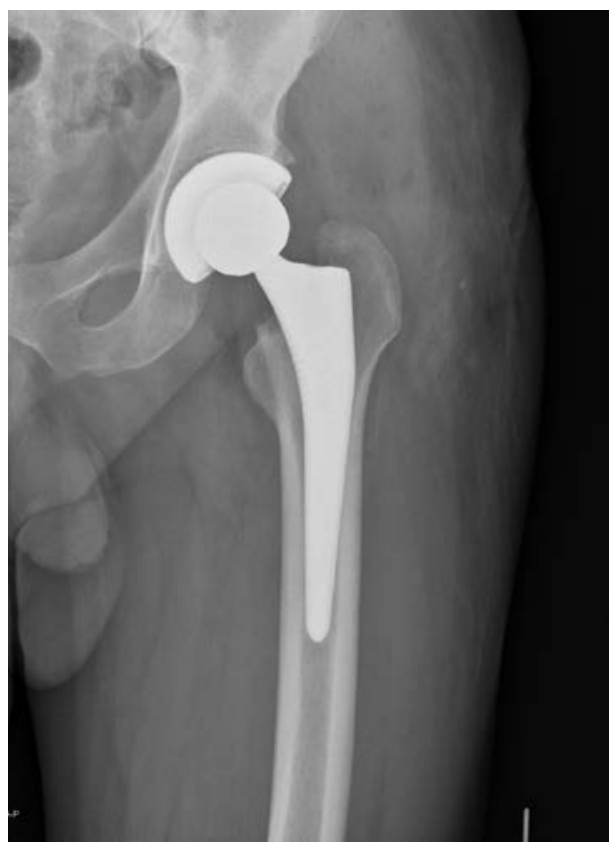

Figure 2. An example of a hip X-ray in a 55-year-old male with non-cement hip replacement

patients since there are some indications that cemented stems are less prone to develop osteolysis; - useful for patients with "stove pipe" type femur, previous fracture, or previous osteotomy since these patients would not be expected to achieve a tight fit which is necessary for ingrowth;

- may be indicated by conditions resulting in poor bone quality such as rheumatoid arthritis, osteoporosis, or Paget's disease;

- due to physical properties, and strong effect of stem size on the cement strains suggests that cemented femoral stems should not be used in heavy patients with small medullary canals that require a small cemented stem.

Bone cement in total hip replacement has several advantages that include: easier affixing of the prosthetic joint components to a bone that is slightly porous from osteoporosis, or a possibility to add a small amount of antibiotic material to the bone cement, helping to decrease the risk of post-surgical infection. However, drawbacks of this method also exist. A breakdown of the cement can cause the artificial joint to come loose, which may prompt the need for another joint replacement surgery (revision surgery). The cement debris can irritate the surrounding soft tissue and cause inflammation. While rare, the cement can enter the bloodstream and end up in the lungs, a condition that can be life-threatening. There is also a complication called bone cement implantation syndrome (BCIS). It is characterized by episodes

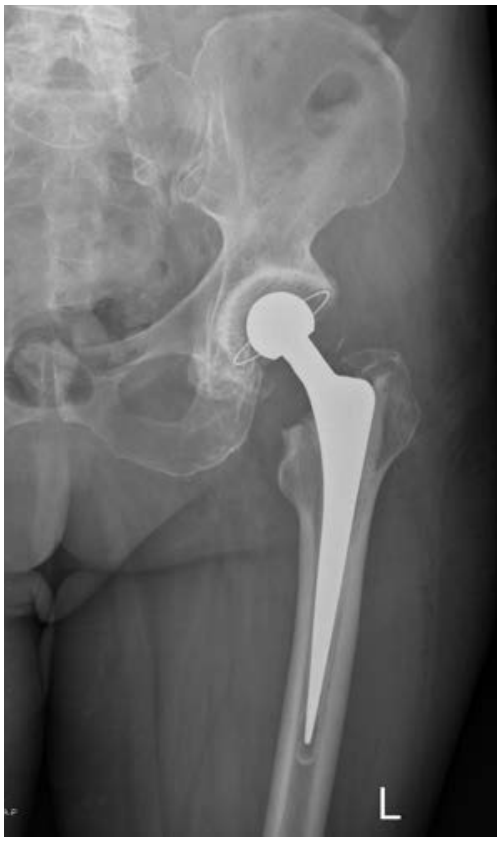

Figure 3. An example of a hip X-ray in an 80-year-old female with hip replacement using cement

of hypoxia and/or hypotension with or without loss of consciousness correlated in time with bone cement application, prosthesis fixation, joint reduction or deflation of tourniquet during cement arthroplasty. It is linked with perioperative morbidity and mortality. The highest risk is related with hip arthroplasty and it is lower in knee arthroplasty and vertebroplasty [11]. The incidence of BCIS is not known as there is no universal definition of the syndrome. According to available data based on non-uniform definitions of $\mathrm{BCIS}$, the occurrence of the syndrome is estimated to occur in about $20 \%$ of cases and mortality secondary to BCIS is about $0.1 \%$ [12].

In our study, we showed that in patients, in whom the use of cement during total hip replacement was necessary, the SBP values were significantly higher before and after the surgery than in patients in whom the cement wasn't used. Prevalence of arterial hypertension was also higher in patients in whom the cement was used. These findings are partially attributable to the higher mean age of the group with cement use. However, we must also keep in mind that patients with the use of cement had significantly lower haemoglobin concentration, haematocrit, $\mathrm{MCHC}$, and significantly higher urea and creatinine levels. Those factors, even despite the older age of patients are risk factors for perioperative complications in hip replacement. It was recently shown that SBP values in the perioperative period are the good predictor of myocardial injury after surgery [13]. While 
hip replacement patients are at high cardiovascular risk [14], it is important to remember that careful management and risk stratification is important in this group, also considering the risk of BCIS.

In conclusion, patients with hip replacement using cement have higher SBP values before, and after the surgery than patients in whom cement wasn't used. These patients are also at higher cardiovascular risk, therefore careful management and risk assessment is especially important in patients receiving the total hip replacement with the use of cement.

\section{References}

1. Kristensen S.D., Knuuti J., Saraste A. et al.; Authors/Task Force Members. 2014 ESC/ESA Guidelines on non-cardiac surgery: cardiovascular assessment and management: The Joint Task Force on non-cardiac surgery: cardiovascular assessment and management of the European Society of Cardiology (ESC) and the European Society of Anaesthesiology (ESA). Eur. Heart J. 2014; 35: 2383-23431.

2. Macellari F., Paciaroni M., Agnelli G., Caso V. Peri-operative stroke risk in nonvascular surgery. Cerebrovasc. Dis. 2012; 34: 175-181.

3. Legosz P., Szymanski F.M., Rys A. et al. Epidemiology of arterial hypertension in patients scheduled for elective hip replacement. Arterial Hypertension. Arterial Hypertens. 2016; 20: 11-15.
4. Wiltse L.L., Hall R.H. Experimental studies regarding the possible use of self-curing acrylic in orthopaedic surgery. J. Bone Joint Surg. Am. 1957; 39-A: 961-972.

5. Mancia G., Fagard R., Narkiewicz K. et al:; 2013 ESH/ESC guidelines for the management of arterial hypertension: the Task Force for the Management of Arterial Hypertension of the European Society of Hypertension (ESH) and of the European Society of Cardiology (ESC). Eur. Heart J. 2013; 34: 2159-2219.

6. Feith R. Side-effects of acrylic cement implanted into bone. A histologi$\mathrm{cal}$, (micro)angiographic, fluorescence-microscopic and autoradiog-aphic study in the rabbit femur. Acta Orthop. Scand. Suppl. 1975: 161:3-136.

7. Kusy R.P. Characterization of self-curing acrylic bone cements. J. Biomed. Mater Res. 1978; 12: 271-305.

8. Wiltse L.L., Hall R.H. Experimental studies regarding the possible use of self-curing acrylic in orthopaedic surgery. J. Bone Joint. Surg. Am. 1957; 39-A: 961-972.

9. Lai P.L., Chen L.H., Chen W.J. et al. Chemical and physical properties of bone cement for vertebroplasty. Biomed J. 2013;36: 162-167.

10. Kuhn K.-D. Bone Cementes. Springer, Germany 2000.

11. Donaldson A.J., Thomson H.E., Harper N.J., Kenny N.W. Bone cement implantation syndrome. Brit. J. Anaesthesia 2009; 102: 12-22.

12. Griffiths R., White S., Moppett I. et al. Safety guideline: reducing the risk from cemented hemiarthroplasty for hip fracture 2015: Association of Anaesthetists of Great Britain and Ireland British Orthopaedic Association British Geriatric Society. Anaesthesia 2015; 70: 623-626.

13. Belen E., Ozal E., Bayyigit A., Gunaydın S., Helvacı A. Interarm systolic blood pressure difference is associated with myocardial injury after noncardiac surgery. Kardiol. Pol. 2016; 74: 674-680.

14. Legosz P., Kotkowski M., Platek A.E. et al. Assessment of cardiovascular risk in patients undergoing total joint alloplasty - the CRASH-JOINT study. Kardiol Pol. 2016 [Article in press]. 